\title{
Sensor-based supporting mobile system Parkinson disease clinical tests utilising biomedical and RFID technologies
}

\author{
Mariusz Chmielewski ${ }^{1, \mathrm{a}}$, Michat Nowotarski ${ }^{1, \mathrm{~b}}$ \\ ${ }^{1}$ Military University of Technology, Cybernetics Faculty, gen. S. Kaliski Street, Warsaw, Poland
}

\begin{abstract}
This paper discusses method and tool for assisting clinical tests of pharmaceutical drugs utilising sensors and mobile technologies. Emerging sensor and mobile technologies deliver new opportunities to gather and process medical data. Presented analytical approach implements such observations and delivers new, convenient means for remote patient monitoring. Clinical tests are highly specialised process requiring methodology and tools to support such research. Currently available methods rely mostly on analogue approach (booklets), requiring the clinical test participant to fill in health state daily. Such approach often can be biased by unpunctual, not precise reporting. The mobile device can support this process by automatic scheduling and recording an actual time of reports and most of all it can record the inertial and biometric sensor data during the survey process. Presented analytical method (tremors recognition) and mobile tool offers consistent approach to clinical test assistance transforming and Android smartphone into remote reporting and notification tool. The tool offers additionally features for sensor based diagnostics support for PD tremor recognition as well as specific clonic and tonic symptoms (dedicated for further system extensions towards epilepsy). Capabilities of the system delivers also RFID mechanisms for efficient on-site clinical test authorisation and configuration. This feature simplifies application installation and automatic set-up considering the participant, clinical test configuration, schedule, smartphone and sensor data. Such a composition delivers convenient and reliable tool which can assist patients and medical staff during the process objectifying the clinical tests results and helping to ensure good quality of the data, quickly available and easily accessible.
\end{abstract}

\section{Introduction and main concept}

The main idea presented in the work is to utilise handhelds and wearable biometric and inertial sensors in order to assist clinical tests and medical examinations [1][7][8]. Such an approach can deliver promising results in order to obtain objective data during massively executed clinical tests. The tool can also lower costs of conducting and analysing results of clinical tests, as the logistics of the tests can be simplified. Presented method, algorithms and its implementation delivers handheld application and a sensor armband, which are managed by the central server service. The central server service can be further integrated with any SOA infrastructure delivered by clinical test provider. Such construct is flexible easily configurable and can be deployed as a cloud services to support scalability.

The use of accelerometer and/or electromyography in neurological diseases treatment were mentioned in various publications. There were discussed ideas to monitor Parkinson's disease with the smartphone [2] or to provide automatic detection of tremors and dyskinesias with accelerometer and electromyographic signal of specified monitoring device [3]. Idea of use an accelerometer during detection of epilepsy's seizures were mentioned in [4][5][6]. What is more, since 2012

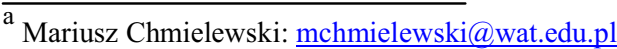

${ }^{\mathrm{b}}$ Michał Nowotarski: michal.nowotarski@wat.edu.pl
Military University of Technology has developed several sensor based solutions for neurological disease assistance and monitoring - SENSE [7], PATRON [8] utilising smartphone's inertial sensors proprietary wireless biometric sensors.

\section{Tool for assisting clinical tests}

PATRON [8] (smartphone-based mobile system utilising wearable sensors) offers innovative approach of Parkinson's Disease symptoms examination delivered through application of myography and inertial sensors integrated with handheld devices. The utilisation of sensor-assisted diagnostics of tremors and motor skills, delivers new opportunities for neurological research especially in the domain of effective drug usage and clinical testing of pharmaceutical therapies. The developed hardware, the PATRON multi-sensor is first fully mobile, wireless sensor fusing several channels of data gathered from surface electromyography, mechanomyography, 3-axis inertial sensor and magnetometer. The sensor can be seamlessly attached to a forearm or calf in order to remotely gather, filter and transfer the data to a handheld. The multistage DSP analysis of the data is performed in real time, delivering 
data of tremors frequency and magnitude, the intensity of muscle tensions and the orientation of the sensor. Fused data describing the neurological examination is than stored, aggregated with the time and location and uploaded to the reporting server. The mobile application delivers two main functionalities ad-hoc tremor examination and on-demand clinical test surveying. Therefore, the application can be used for examining, the tremor intensity and limb movement fluency, evaluating the Parkinson's Disease on-off fluctuations and dyskinesia. System supports the PD patient by estimating current health state and in consequence providing recommendation for medicine usage. PATRON can also be used as a clinical test support toolkit for researchers and pharmaceutical companies guiding patients through the complete process of reporting the drug effectiveness. Sensor-based tests complement the patient's surveys, delivering objective information about the patient's health state therefore evaluating the effectiveness of a drug and applied treatment. The system offers also a server based reporting services which serve as a clinical tests warehouse. Web portal visualizes the clinical test process organizes the data and evaluates the characteristics of the medicine effectiveness [13][17]. PATRON server has been deployed on Microsoft Azure Cloud environment, which provides high-scalability and security characteristics. Web application can be accessed by the researchers, medical staff and pharmaceutical companies in order to inspect and monitor current state of clinical test process.

\section{Utilisation of inertial myography sensors}

The MYO armband is a gesture and movement control device. Its purpose is to receive signals during hand's movement and gestures. The device contains eight EMG sensors (device segments) detecting muscle tension with sample rate $200 \mathrm{~Hz}$ and accelerometer, gyroscope and magnetometer inside built-in IMU with sample rate $50 \mathrm{~Hz}$ (Invensense MPU-9150 9-dof motion sensor). It is controlled by the Free-scale Kinetis Cortex M4 $120 \mathrm{MHz}$ processor and communication is realized with Micro USB port or Bluetooth LE protocol. Armband can be expandable between 7.5 - 13 inches (19 - $34 \mathrm{~cm}$ ) forearm circumference and weights 93 grams [9][10].

The vendor has provided SDKs dedicated for various mobile platforms and capabilities. For example, the iOS SDK contains methods providing ability to read data from EMG sensors, while the Android SDK lacks such features. According to [11], sensors of the Myo have the following characteristics:

- electromyograph - unit of measurement: $\mathrm{mV}$, range: $+/-0.45 \mathrm{mV}$,

- accelerometer - unit of measurement: g, range: +/- 16 g,

- gyroscope - unit of measurement: $\%$, range: $+/-2000$

$\%$ s,

- position sensor - unit of measurement: quaternion.
The manufacturer claims that sensor's battery should last a whole day of continuous use (which is one of crucial requirements for the clinical test assistance). For the band in a sleep mode, disconnected from any devices, expected operating time is about a week. There are two batteries inside, with a capacity of $260 \mathrm{mAh}$ each. The communication range via Bluetooth is up to 15 meters. Armband's sensors can work on the leg what can be useful in various diagnostic purposes.

Myo was mentioned before as an device to medical use. It was proposed to help hand-amputees recover functionality using non-invasive method [12]. Its surface electromyography accuracy was compared and defined as similar to more expensive devices.

\section{Clinical test support}

The main purpose of the application is to continuously monitor the patient's condition. Therefore, at a time specified according to the schedule, application displays a notification asking the user to complete a short survey about his health. The schedule can be configured according to the patient's individual diagnostic needs. The survey initially contains the following questions:

- Are You able to perform survey? (Yes, No)

- Rate your state (On, On-Off, Off)

- Do you have dyskinesias? (Yes, No)

- Are these dyskinesias inconvenient?(Yes, No) The list of survey questions can be remotely configured, modifying questions and possible answers - with respect to versioning of entities (required for analytical processing). Additionally the functionality of system delivers advanced scheduling of surveys depending on the day and night cycle and the intensity of the disease. These questions were developed on the basis of questionnaires that are presented to patients to assess the effectiveness of their drugs. The application can record also drugs intake (time and dose) reported for further correlation analysis.

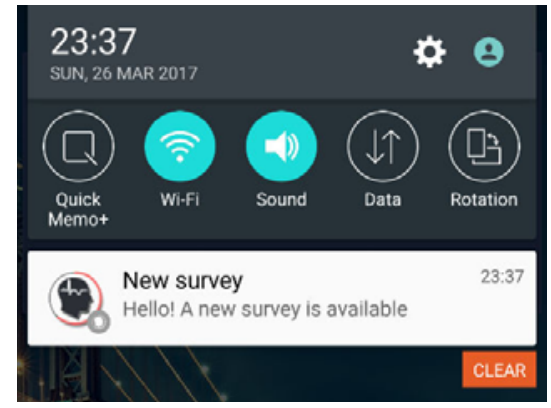

Fig. 1. New survey to fill notification displayed in the smartphone's notification toolbar

User-supplied responses are stored in the device memory and optionally sent to reporting-acquisition service. Depending on the user abilities and handheld capability the process manager can choose the preferred form of report gathering (on-site and remote method). During the survey the device and additional sensors 
placed on the participant's body record answers and sensor signals (inertial and EMG). Each and every survey can take from 30-50 seconds in order to record and store statistically significant data. In addition, the application reminds the patient to take his medications and to schedule a consultation visit. The variety of reported events can be further extended as the reported data can be used as a form of detailed treatment assessment report - correlating patient's comfort and health state with environment and psychiatric state. Such data are stored automatically utilising built-in sensors or manually reported events on which the server inference system is able to recognise correspondences. The inference system has been designed as an ontology with stored rules performing patient health state data classification.

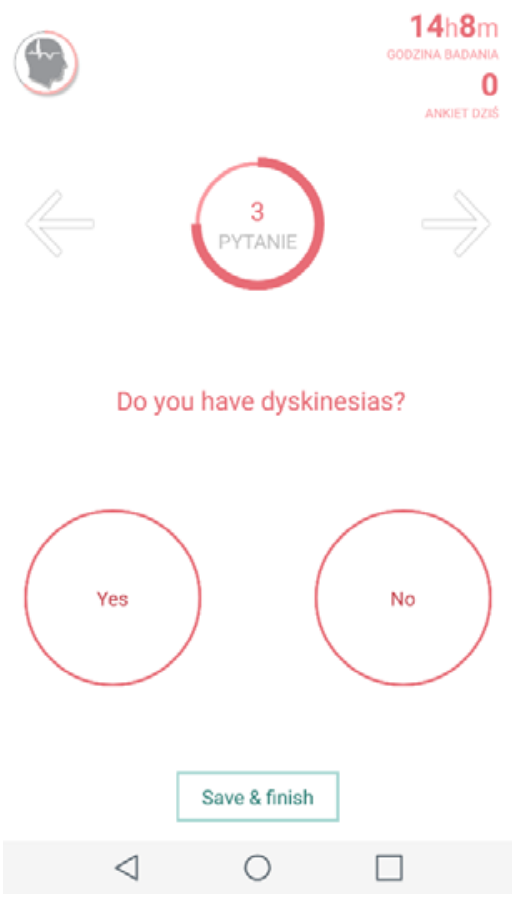

Fig. 2. Patron Droid survey screen, question number 3

The application generates all notifications (with proper vibration signalling and survey presentation) about pending survey requests. All requests store time of generation and time of recorded answers which is further used by the analytical engine. System's mechanisms try to encourage the user to constantly and regularly report good quality data rather than penalty any unwanted and faulty behaviour. To identify and overcome such data, the server side provides mechanism tagging such surveys, leaving the final decision to clinical test process manager [13][17]. The system delivers also conventional application mode, in which the user can request sensor assisted medical diagnostics, record and evaluate current health state. Patron delivers also mechanisms to identify some neurological symptoms of Parkinson's Disease using assessment of symptoms during diagnostic exercises (the flow of forearm movement, etc.). The clinical test participant is able to self-start an examination procedure which will be guided by the application using voice generated notifications. Before the test start, the user has to choose the basic configuration, such as utilised sensors and evaluated body (arm) side. The medical examination mechanisms starts to guide the user through the process of properly placing the sensors, preparing for examination, the examination itself and its assessment. The data gathered in the whole process are stored in form of CSV and binary files for further extraction and processing. This application mode, can be used to evaluated current participant state on-demand or to confirm possible tremors and dyskinesias.

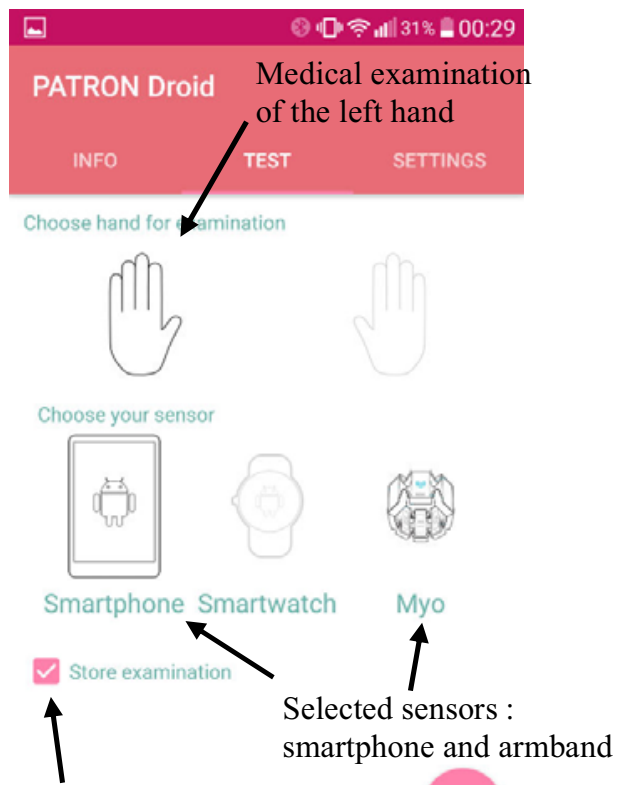

Save data to file

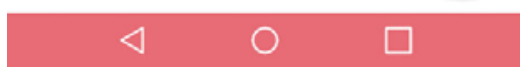

Fig. 3. Patron Droid examination's configuration main screen. Selected configuration is: medical examination of the left hand, use smartphone and armband sensor and save data to a CSV file

Before the test, the paired band is searched and connected. The user can see a screen presenting the selected hand on which he choose to test and status of sensors that he has chosen. Then the user has time to prepare for the test. If he is ready, he has to press the start button. The countdown occurs, and then the test begins.

The test has a specified time set by the user. At the top of the examination screen is a clock counts the remaining time to complete the test. Below, real-time charts for each signal type and for each sensor are drawn with their current values displayed as a number. During the test, all signals received from the sensors are passed to the algorithm receiver. At the same time, if the user has marked the option of saving to a file, the data is passed to the file writer component.

The test continues until the user-defined time has elapsed. If this is a one hand test, the test is completed. If 
the user set up a test for both hands, he can review the test for the selected hand and then he is asked to change his hand. After making the change, he has to press the start button again and the test is carried out for the second hand.

There is presented a screen of the application during performing an examination on the Fig. 4.

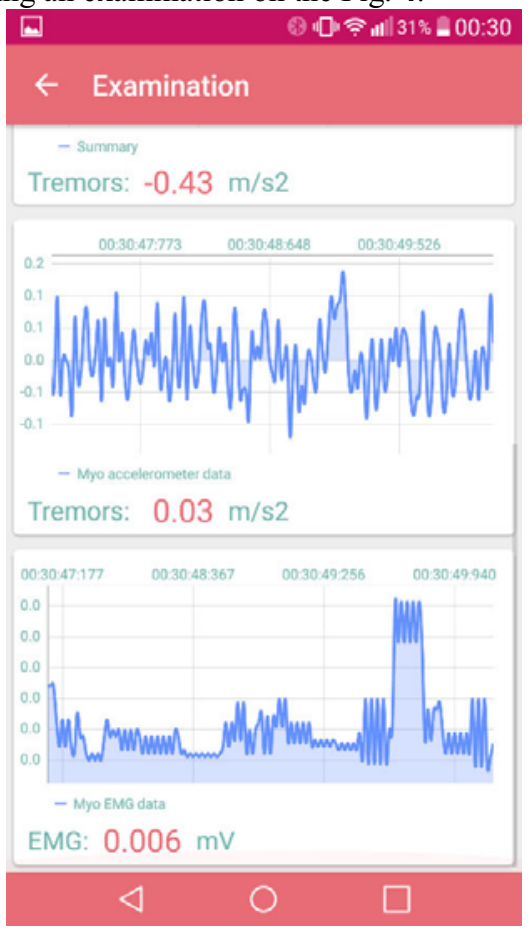

Fig. 4. Patron Droid examination screen

During the examination, the algorithm determines the individual parameters for specified time frame. At the end of the operation time, the reasoning is performed on the obtained parameters, and the result of the test is displayed to the user.

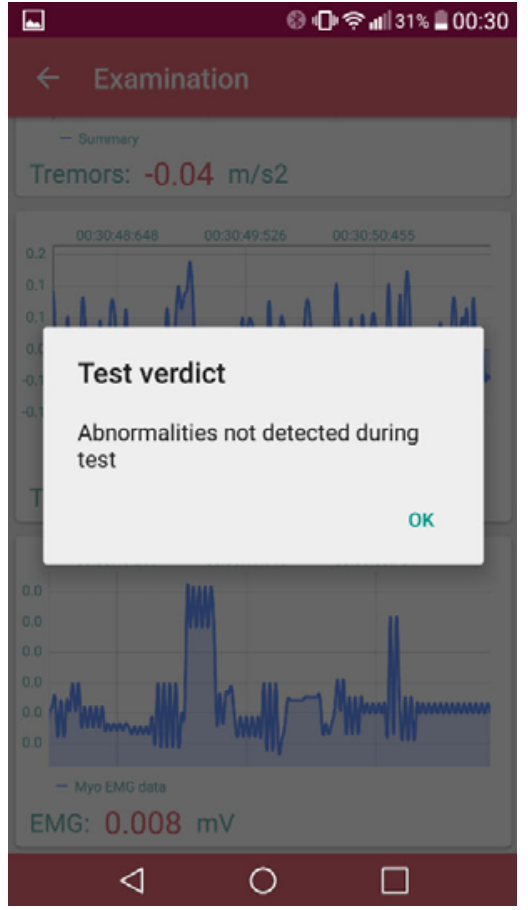

Fig. 5. Patron Droid examination result screen

If user chose to save test data, there is a file created in a device's memory. It holds the user data, test configuration and received data. Below is an example of a CSV file's fragment with data obtained during an examination.

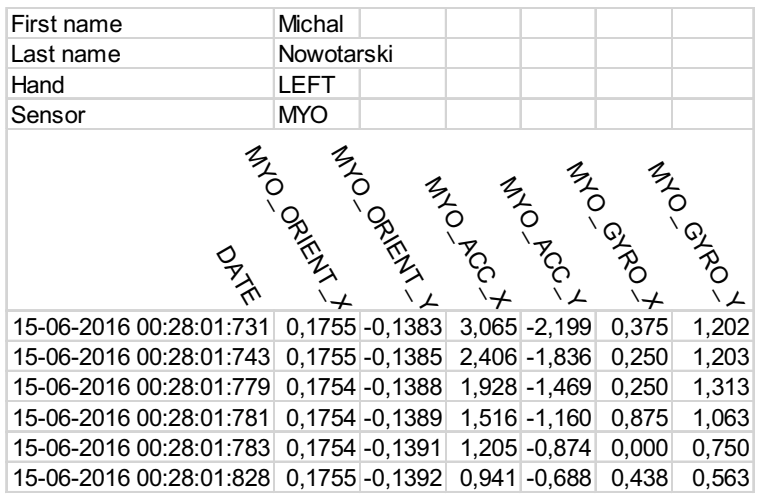

Fig. 6. Part of Patron Droid examination's data saved file

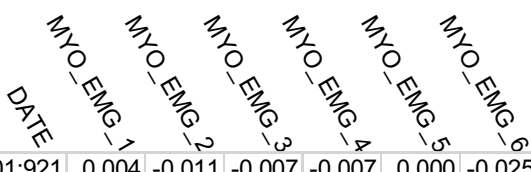

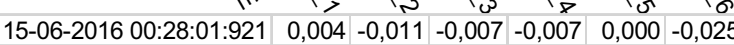

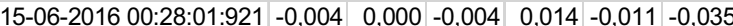

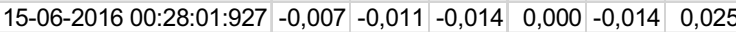
\begin{tabular}{|l|l|l|l|l|l|l|l|l|l|l|}
\hline $15-06-2016$ & $00: 28: 01: 927$ & 0,000 & $-0,014$ & $-0,007$ & 0,007 & $-0,011$ & $-0,028$ \\
\hline
\end{tabular} 15-06-2016 00:28:01:932 $-0,007 \quad-0,011 \quad-0,014 \quad 0,000 \quad-0,014 \quad 0,025$

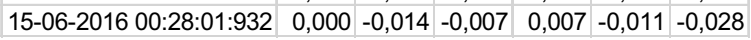

Fig. 7. Part of Patron Droid examination's data saved file 
Before performing the first examination, user has to configure the application properly. In the "settings" tab user has to fill his personal data and configure the examination. User can choose hand configuration to test (one hand or both hands), examination time and set thresholds necessary for the proper work of the algorithm. At the tab, user can also pair his armband sensor with the application, what is required to use it during examinations.

\subsection{PD Examination algorithm}

The idea was to use both, an accelerometer and an electromyographic signals during diseases detection. It was targeted to detect symptoms of the Parkinson's disease and epilepsy. Both are related to dyskinesias but with slightly different intensification. The Parkinson's disease is associated with light limbs movements without increased muscle tension while the epilepsy is related to muscle tension without body movement (tonic phase) or tensioning and relaxing muscles with rapid movement (clonic phase). On the basis of the research it has been found that it is worth to measure the correlation between test results of both hands.

First step of algorithm's use is to set limit values for electromyograph and accelerometer data in the "settings" tab. These are values in $\mathrm{mV}$ or $\mathrm{m} / \mathrm{s}^{2}$ which are thresholds for exceeded limit value counters. They have to be float values, greater than zero.

Before an examination start, user has to set up a configuration:

- one or both hand test,

- which hand user want to test,

- use of smartphone and/or smartwatch and/or armband sensors.

The algorithm receives data from sensors during examination and calculate parameters separately for both hands (left, right) and every enabled sensor (ACC, EMG). Every five seconds the following parameters: minimum, maximum, deviation value and number of samples which exceeded limit values are being calculated.

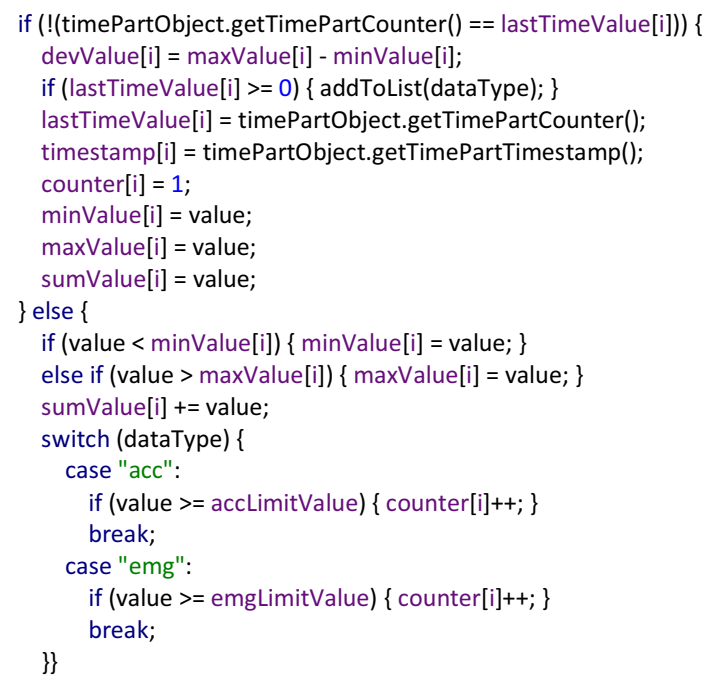

Fig. 8. Code fragment for calculating parameters for a time frame

After the examination's end, these parameters are checked: if there is an exceed of values, appropriate flags are set to true.

Last step of the algorithm is flags check. They are compared to the test's configuration and based on these information, the final result of the examination is being raised.

As was mentioned before, different measurement devices have various sensors: smartphone and smartwatch have accelerometer, gyroscope and orientation sensor, while the armband has all of the above with an additional electromyographic sensor. It causes that different devices can provide results of different accuracy. What is more, both hands examination can provide more specific data than one hand examination.

As it was mentioned before, the test result is being determined on the basis of a comparison of flags set during the processing and configuring of the test. Based on the examination configuration:

- For all configurations user can receive result "no abnormalities detected".

- For one hand without electromyographic data it could be "Parkinson's disease or epilepsy suspicion", because the result is based only on the movement signal.

- Testing both hands without the armband sensor, allows the algorithm to distinguish Parkinson's disease suspicion from epilepsy suspicion, because of Parkinson's only one side (hand or leg) trembling.

- with use of the armband sensor, it could detect Parkinson's disease, epilepsy tonic phase or epilepsy clonic phase suspicion based on the ACC and EMG signals. 


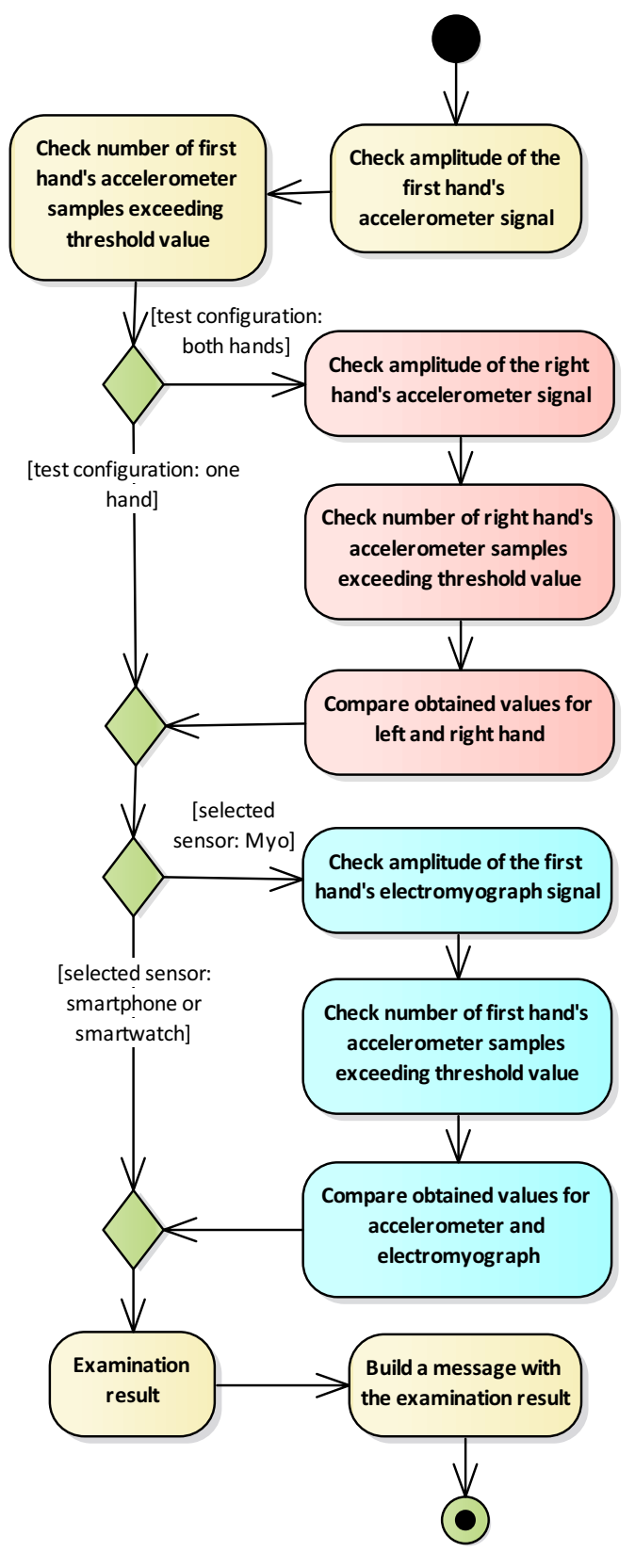

Fig. 9. Flow of the algorithm

Obtained results depend on the occurrence of the problems identified during the examination:

- No abnormalities means there was not set any of the examination flags.

- The Parkinson's disease or the epilepsy suspicion based on hand trembling during examination of one hand without the armband sensor.

- The Parkinson disease suspicion implies that there was hand trembling without muscle tension during examination of one hand with the armband sensor or only one hand trembling during any examination of both hands.
- The epilepsy tonic phase suspicion is based on increased muscle tension without hand trembling during an examination with the armband sensor.

- The epilepsy clonic phase suspicion means that there was variable muscle tension and hand trembling during an examination with armband sensor use.

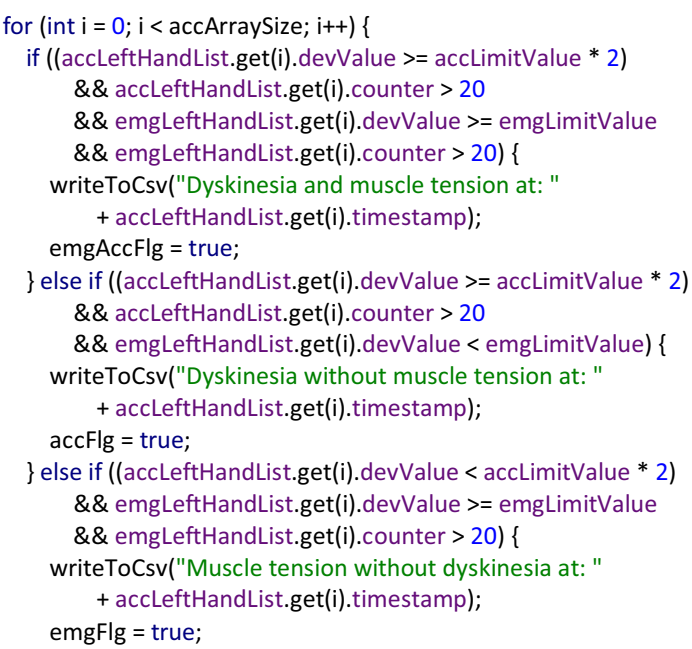

Fig. 10. Code fragment for checking calculated parameters and making a test result

\subsection{Second stage of the algorithm}

The current algorithm is not sufficient for advanced diagnosis purposes, so a plan for its further development has already been prepared. In the next stage the algorithm will be significantly expanded. The diagnosis of Parkinson's disease will use an actigraphy to measure mobility of the limb. In the case of epilepsy, the purpose is to detect particular types of seizures. To achieve this, during the test will be determined muscle activation function and measured intervals between succeeding slopes on the signal record. Based on these data and accelerometer the tonic and clonic phases will be detected. The algorithm will search for similar characteristics as currently (described in 4.1). Atonic seizures will be detected by sensors located on the patient's neck, as a best place to observe their characteristics. This is a sudden momentary stroke of muscle tension along with the following drop on the EMG signal. It will be correlated with patient's behaviour (usually a fall-off) found on the inertial sensor. The algorithm will also detect myoclonic seizures that are characterized by sudden muscle spasms in the electromyographic signal. It is also intended to use the machine learning technique in patterns comparing.

At the moment, software authors do not have large sets of research data yet. At this point the research is done in several dozen attempts on two patients who reported to study.

In addition, research are still conducted to make it possible to identify if obtained sensors data allows to accurately identify conditions in patients' behaviour 
which are needed to diagnose properly. One of the issues is to determine the visibility of Parkinson's tremors in the electromyography record

\subsection{Data receive}

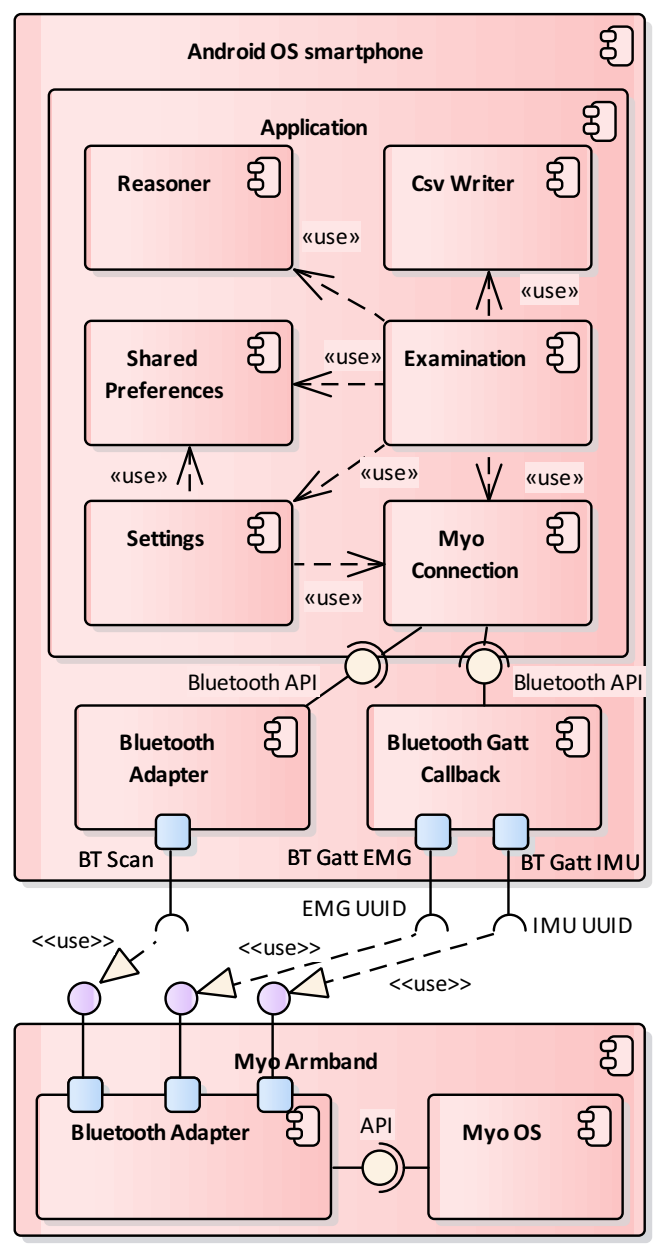

Fig. 11 Component model of the application

The main difficulty was to receive an EMG data on a mobile device straight from the armband due to lack of EMG capture methods in the official Android SDK. The solution was an own implementation of Bluetooth connection, without using the official SDK. There is released file in $\mathrm{C}++$ which describes commands used in Bluetooth communication with the Myo [11]. Having used content of the file, it was possible to write a full connection with the Myo in Java using built-in Android's Bluetooth LE libraries. The connection implementation is based on a Meleap's code shared on the GitHub website ${ }^{\mathrm{a}}$. The connection is being realized by a component called Myo Connection. It contains methods related to establishing and keeping a

\footnotetext{
${ }^{\text {a }}$ https://github.com/meleap/myo_AndoridEMG
}

connection, receiving and parsing a data. It uses android Bluetooth components: the Bluetooth Adapter and the Bluteooth Gatt Callback. The Bluetooth Adapter searches for nearby Bluetooth devices or connect to saved one and passes connection parameters to the Gatt Callback. The Callback registers the application in armband's services (control service, IMU service, EMG service), receives data from them and sends messages to the Myo's control service.

Data received from services is provided as byte data and has to be parsed to appropriate form described in the Myo Bluetooth protocol file. Having parsed data, there is an ability to use it in the algorithm. Data consists of a accelerometer, gyroscope, magnetometer, orientation and electromyographic signal values is processed with appropriate timestamps.

The test execution is implemented as follows:

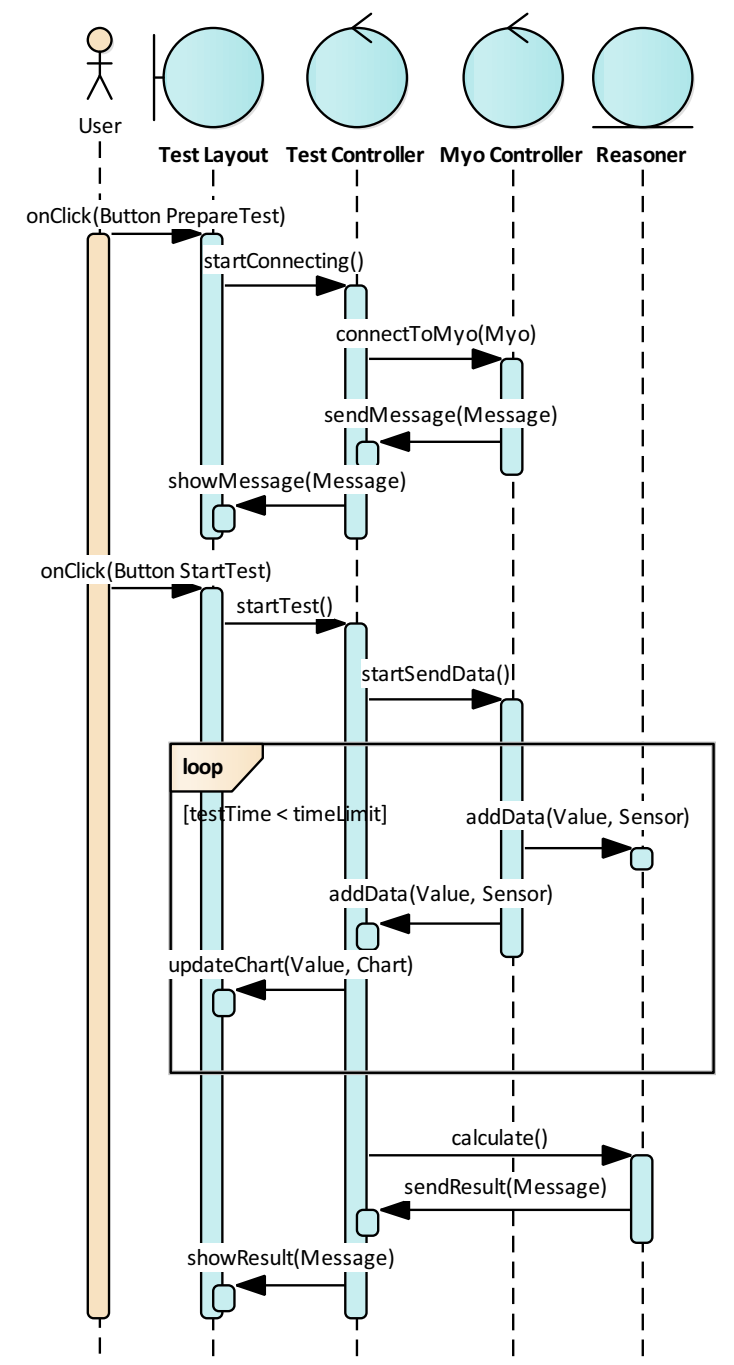

Fig. 12. Sequence diagram of the examination execution

\footnotetext{
public byte[] sendEmgAndImu() \{

byte command_data $=($ byte $) 0 \times 01$

byte payload_data $=$ (byte) 3 ;
} 


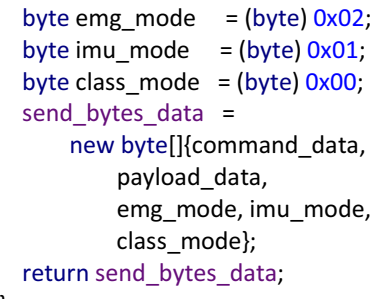

Fig. 13. Code fragment for configuring sensor working mode to send accelerometer, orientation, gyroscope and electromyograph data

\subsection{Restrictions}

To work properly, the algorithm has to be used in a prepared environment. It means some constraints:

- user has to provide valid threshold data for the algorithm,

- the examination must be carried out on a person who is not moving during whole test,

- application cannot be closed or minimized,

- detection of diseases is based on selected symptoms,

- symptoms are consistent with these described before,

- the examination is done with a sensor placed on a hand with disease symptoms.

\section{Experiments and tests}

Tests were made by the application developer and was simulated to cover different examination cases. Every test was made with different hand movement, different muscle tension and the same test configuration. Test were conducted with the armband sensor use. Charts were made based on the data received and saved from sensors during examination. Test verdict is a result presented to user by the application. Limit values was set to $0.8 \mathrm{~m} / \mathrm{s}^{2}$ for an accelerometer and $0.06 \mathrm{mV}$ for an electromyograph.

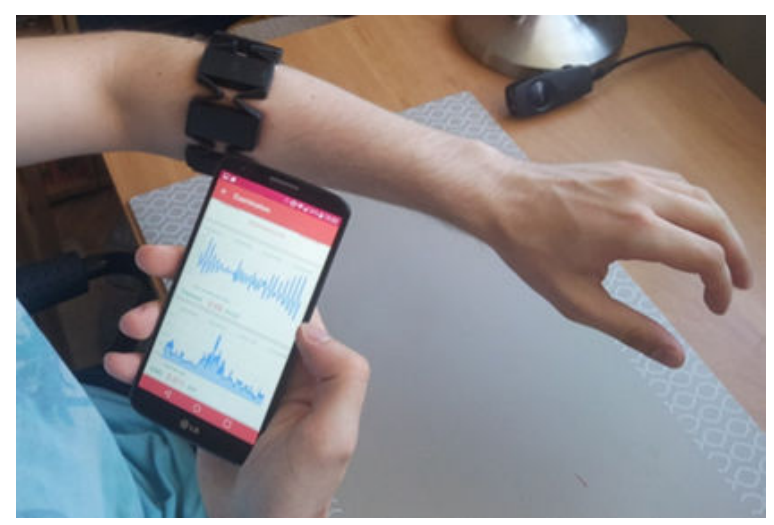

Fig. 14. A sensor test (diagnostics) processed using 2 inertial data sources and EMG - smartphones and MYO biometric sensor

\subsection{Test case 1}

The simulation of a healthy person's behaviour. It means that there is not any excessive handshake or muscle tension. The hand was held stably, without any movement and the accelerometer signal (blue) was maintained throughout the examination at a low level. Low values on the electromyograph chart (orange) indicates that throughout the study muscles were not significantly tightened. That shape of the graph should be achieved for a healthy person. The algorithm did not detect any irregularities in the movement of the hand or muscles tense. Both accelerometer and electromyography parameters were not exceeded enough to set flags to true. Only 3 accelerometer samples have greater value than limit value and none of electromyographic samples was greater than limit. That induced final result: Abnormalities not detected during test.

Table 1. Statistics of the Test case 1

\begin{tabular}{|l|l|l|}
\hline \multicolumn{2}{|l|}{ ACC } & EMG \\
\hline $\begin{array}{l}\text { No. of samples } \\
\begin{array}{l}\text { No. of samples } \\
\text { exceeded }\end{array}\end{array}$ & 1004 & 1002 \\
$\begin{array}{l}\text { values } \\
\text { Minimum value }\end{array}$ & $-0.214 \mathrm{~m} / \mathrm{s}^{2}$ & $0.001 \mathrm{mV}$ \\
$\begin{array}{l}\text { Maximum value } \\
\text { Deviation between } \\
\text { min and max }\end{array}$ & $1.378 \mathrm{~m} / \mathrm{s}^{2}$ & $0.035 \mathrm{mV}$ \\
\hline
\end{tabular}

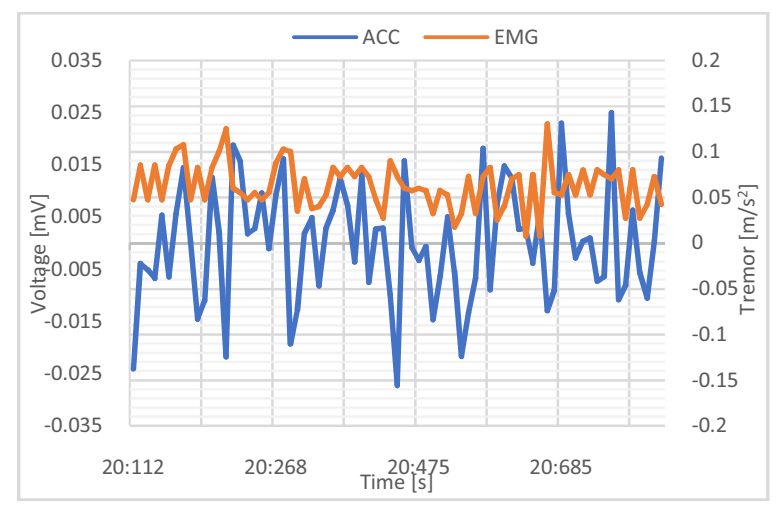

Fig. 15. Graph of extracted 150 samples started at $1000^{\text {th }}$ sample of the Test case 1. Blue line is an accelerometer signal, orange line is an electromyograph signal.

\subsection{Test case 2}

The simulation of a person suffering from the Parkinson's disease. During the test, arm was still raised and entered in a gentle tremor. Muscles were relaxed during whole examination. Compared to the test of a healthy person, a signal from the accelerometer has higher values. It can be seen the effect of delicate hand shake on the value of the received signal. Together with the accelerometer, it is possible to see a slight increase in the value of the electromyographic signal. The algorithm detects handshake without significant change of the muscle tension. Handshake can be observed as 385 
samples exceeded limit values. This amount could not have been a random hand movement, so the 20 exceeded samples in a 5 second time window condition was fulfilled and an accelerometer flag was set to true. The electromyographic signal were not significantly increased as none of samples was greater than threshold values. The algorithm detected the undesired movement of the hand without significant changes in muscle tension so the result was suspicion of the Parkinson's disease.

Table 2. Statistics of the Test case 2

\begin{tabular}{|lll|}
\hline \multicolumn{2}{|l|}{ ACC } & EMG \\
\hline $\begin{array}{l}\text { No. of samples } \\
\text { No. of samples } \\
\text { exceeded }\end{array}$ limit & 982 & 970 \\
$\begin{array}{l}\text { values } \\
\text { Minimum value }\end{array}$ & $-2.321 \mathrm{~m} / \mathrm{s}^{2}$ & $0.003 \mathrm{mV}$ \\
$\begin{array}{l}\text { Maximum value } \\
\text { Deviation between } \\
\text { min and max }\end{array}$ & $1.962 \mathrm{~m} / \mathrm{s}^{2}$ & $0.059 \mathrm{mV}$ \\
\hline
\end{tabular}

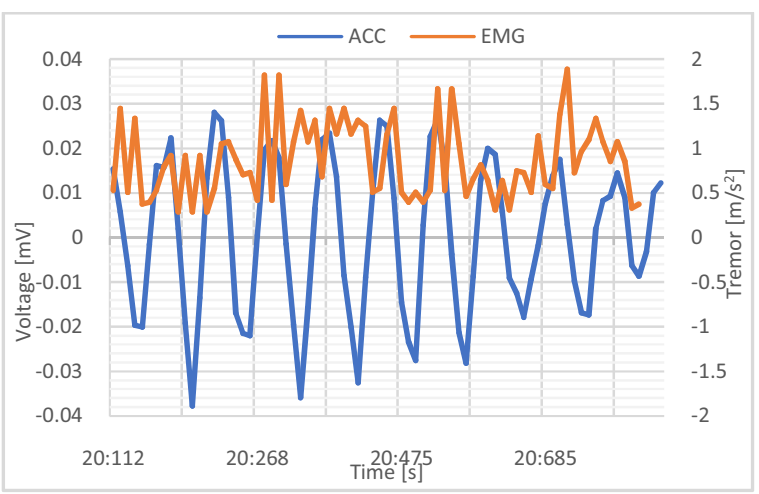

Fig. 16. Graph of extracted 150 samples started at $1000^{\text {th }}$ sample of the Test case 2. Blue line is an accelerometer signal, orange line is an electromyograph signal.

\subsection{Test case 3}

The simulation of a person in the tonic phase of epilepsy. Hand was held in one position. To keep muscles tightened, a fist was clenched. Muscles were tensed all the time with a full strength. During the examination there was not any hand shake. The signal from the accelerometer confirms the lack of hand movement during the test. However, the flow of the electromyographic signal clearly indicates a significant and constant increase in muscle tension. After muscles having tensed, this state was maintained for about 8 seconds and then muscles were relaxed. The algorithm detected a strong muscle tension with the stillness of the hand. It is based on nearly none of accelerometer samples exceeded threshold value. It caused to keep accelerometer flag set to false. With nearly 450 electromyograph samples with values greater than limit, 20 samples in a 5 second time window condition was fulfilled and an electromyograph flag become true. That led to conclusion as the suspected was the tonic phase of epilepsy.

Table 3. Statistics of the Test case 3

\begin{tabular}{|l|l|l|}
\hline \multicolumn{2}{|l|}{ ACC } & EMG \\
$\begin{array}{l}\text { No. of samples } \\
\begin{array}{l}\text { No. of samples } \\
\text { exceeded limit }\end{array}\end{array}$ & 979 & 970 \\
$\begin{array}{l}\text { values } \\
\text { Minimum value }\end{array}$ & 445 \\
$\begin{array}{l}\text { Maximum value } \\
\begin{array}{l}\text { Deviation between } \\
\text { min and max }\end{array}\end{array}$ & $-0.990 \mathrm{~m} / \mathrm{s}^{2}$ & $0.077 \mathrm{~m} / \mathrm{s}^{2}$ \\
\hline
\end{tabular}

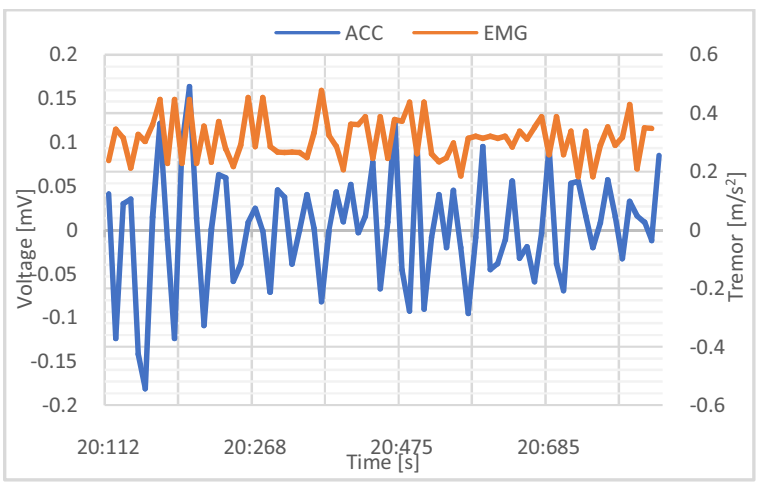

Fig. 17. Graph of extracted 150 samples started at $1000^{\text {th }}$ sample of the Test case 3. Blue line is an accelerometer signal, orange line is an electromyograph signal.

\section{$5.4 \quad$ Test case 4}

The simulation of a person in the clonic phase of epilepsy. During the test execution, there were performed some rapid hand movements with tensioning and relaxing of muscles. The graph shows the movements of the hand on the accelerometer signal and tensioning and relaxing muscles on the record of the electromyograph signal. Algorithm detected in this case rapid hand movements with significant changes in muscle tension. Both accelerometer and electromyograph signals were significantly greater than thresholds on a large number of samples. Both flags, for accelerometer and electromyograph were set to true due to its met requirements of 20 samples exceeded limit values in a 5 second time window. Conclusion for the situation was: epilepsy clonic phase suspicion.

Table 4. Statistics of the Test case 4

\begin{tabular}{|c|c|c|}
\hline & ACC & EMG \\
\hline No. of samples & 977 & 973 \\
\hline $\begin{array}{lr}\text { No. of } & \text { samples } \\
\text { exceeded } & \text { limit } \\
\text { values } & \end{array}$ & 573 & 589 \\
\hline Minimum value & $-11.680 \mathrm{~m} / \mathrm{s}^{2}$ & $0.004 \mathrm{mV}$ \\
\hline Maximum value & $19.484 \mathrm{~m} / \mathrm{s}^{2}$ & $0.296 \mathrm{mV}$ \\
\hline Deviation & $31.164 \mathrm{~m} / \mathrm{s}^{2}$ & $0.293 \mathrm{mV}$ \\
\hline
\end{tabular}


min and max

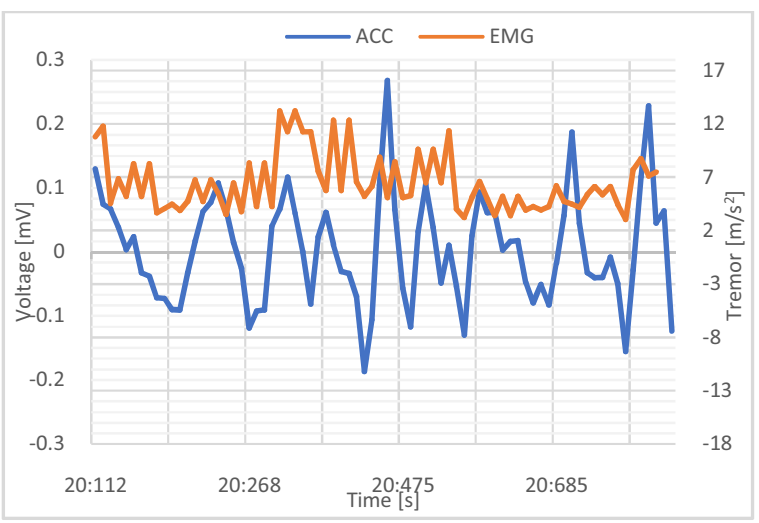

Fig. 18. Graph of extracted 150 samples started at $1000^{\text {th }}$ sample of the Test case 4. Blue line is an accelerometer signal, orange line is an electromyograph signal.

\section{Summary}

Presented in the paper method and tool offers proprietary algorithms to support acquisition, analysis and medical diagnostics of selected neurological symptoms. The mechanisms cannot replace the professional help and detailed medical diagnostics. During system tests implemented algorithms proved their usefulness especially in the range of tremors intensity evaluation. Such information can be used for PD's drug usage forecast, as the pharmaceuticals have specific action time.

many but can assist clinical tests, but can be helpful when Application of sensors and complex algorithms requires specific configuration and environment preparation (described in 4.4.).

Results received during tests were consisted with theoretical expectations. Signals that were picked up by the application from the armband sensor reflected the behaviour of the tested person. With increased muscle tension, electromyographic signal significantly increased its value and vary depending upon the strength of the tension. A similar situation was observed in the accelerometer signal. Values were changing with the movement of the hand and band sensor obtained values corresponded to smartphone accelerometer signal. This allows to state that data received from the band were correct.

In addition, results of tests were consistent with the assumptions. On the basis of signals' characteristics, algorithm properly diagnosed diseases. This allows to evaluate that produced diagnostic tool has been implemented correctly. It is a good base for further development or research related to the diagnosis of neurological disorders with mobile devices. RFID technology [15][16] proved to be useful channel for clinical test initial configuration and on-site clinical test collection mechanisms. It has been used to authenticate and preconfigure transmission channel while maintaining patient's clinical records anonymous.
Further extensions of RFID usage for automatic drug dose configuration and drug repository maintenance.

What is more, the way the application was built allows to expand it with another measurement devices or to implement more complex mechanisms for inferring and analysing user behaviour.

The system has been officially deployed after successful beta tests on population of 10 patients which have been using the application for at least 4 months. At the time of paper submission a formal acceptance of system and sensor clinical tests have been approved.

\section{References}

1. M. Nowotarski: Opracowanie algorytmów decyzyjnych wykorzystujących wybrane sygnały inercjalne oraz biomedyczne do identyfikacji zaburzeń neurologicznych oraz oprogramowania mobilnego na platformę Android, master's thesis, M. Chmielewski (supervisor), MUT (2016),

2. R. LeMoyne: Wearable and wireless accelerometer systems for monitoring Parkinson's disease patients-A perspective review, Advances in Parkinson's Disease, 2, 113-115 (2013)

3. S.H. Roy, B.T. Cole, L.D. Gilmore, C.J. De Luca, C.A. Thomas, M.M. Saint-Hilaire, S.H. Nawab: High-Resolution Tracking of Motor Disorders in Parkinson's Disease During Unconstrained Activity, Movement Disorders, 28, 1080-1087 (2013)

4. S. Beniczky, T. Polster, T. W. Kjaer, H. Hjalgrim: Detection of generalized tonic-clonic seizures by a wireless wrist accelerometer: A prospective, multicenter study, Epilepsia, 54, 58-61 (2013)

5. M.M. Velez, R.S. Fisher, V. Bartlett, S. Le: Tracking generalized tonic-clonic seizures with a wrist accelerometer linked to an online database, Seizure, 39, 13-18 (2016)

6. J.R. Villar, M. Menendez, J. Sedano, E. de la Cal, V.M. Gonzalez: Analyzing accelerometer data for epilepsy episode recognition, 10th International Conference on Soft Computing Models in Industrial and Environmental Applications. Advances in Intelligent Systems and Computing, 368, 39-48, Springer, Cham (2015)

7. SENSE project Wiki: http://uranus.wat.edu.pl:8808/wiki/index.php/ SENSE (access: 2017.05.05)

8. PATRON project wiki: http://uranus.wat.edu.pl:8808/wiki/index.php/ PATRON (access: 2017.05.05)

9. Myo tech spec: https://www.myo.com/techspecs

10. B. Stern: Inside Myo, adafruit.com (2016): https://learn.adafruit.com/myo-armbandteardown/inside-myo

11. Myo Bluetooth Low Energy specification file: https://github.com/thalmiclabs/myobluetooth/blob/master/myohw.h

12. M. Abduo, M. Galster: Myo Gesture Control Armband for Medical Applications, University of Canterbury (2015) 
13. R. Hoffmann, M. Kiedrowicz, J. Stanik, Risk management system as the basic paradigm of the information security management system in an organization, 20th International Conference on Circuits, Systems, Communications and Computers, MATEC Web of Conferences, Cedex A: E D P Sciences, vol. 76, (2016).

14. M. Kiedrowicz, T. Nowicki, R. Waszkowski, Z. Wesolowski, and K. Worwa, Method for assessing software reliability of the document management system using the RFID technology, 20th International Conference on Circuits, Systems, Communications and Computers, MATEC Web of Conferences, Cedex A: E D P Sciences, vol. 76, (2016).

15. M. Kiedrowicz, Location with the use of the RFID and GPS technologies - opportunities and threats, GIS ODYSSEY 2016, pp. 122-128, (2016).

16. M. Kiedrowicz, Objects identification in the informations models used by information systems, GIS ODYSSEY 2016, pp. 129-136, (2016).

17. M. Kiedrowicz, T. Nowicki, R. Waszkowski, Business process data flow between automated and human tasks, 3rd International Conference on Social Science (ICSS 2016) December 9-11 2016, pp. 471477, (2016). 